\title{
BMJ Open Nurses' experiences of caring for people with COVID-19 in Hong Kong: a qualitative enquiry
}

\author{
Janita Pak Chun Chau (D) , ${ }^{1}$ Suzanne Hoi Shan Lo (D) , ${ }^{1}$ Ravneet Saran, ${ }^{1}$ \\ Claudia Ho Yau Leung, ${ }^{1}$ Simon Kwun Yu Lam, ${ }^{1}$ David R Thompson ${ }^{2}$
}

To cite: Chau JPC, Lo SHS, Saran R, et al. Nurses' experiences of caring for people with COVID-19 in Hong Kong: a qualitative enquiry. BMJ Open 2021;11:e052683. doi:10.1136/ bmjopen-2021-052683

- Prepublication history and additional supplemental material for this paper are available online. To view these files, please visit the journal online. (http://dx.doi.org/10.1136/ bmjopen-2021-052683)

Received 23 April 2021 Accepted 11 August 2021
Check for updates

(C) Author(s) (or their employer(s)) 2021. Re-use permitted under CC BY-NC. No commercial re-use. See rights and permissions. Published by BMJ.

${ }^{1}$ The Nethersole School of Nursing, Faculty of Medicine, The Chinese University of Hong Kong, Hong Kong, China

${ }^{2}$ School of Nursing and

Midwifery, Queen's University Belfast, Belfast, UK

Correspondence to Dr Janita Pak Chun Chau; janitachau@cuhk.edu.hk

\section{ABSTRACT}

Objectives Nurses are the largest group of healthcare workers on the front line of efforts to control the COVID-19 pandemic. An understanding of their nursing experiences, the challenges they encountered and the strategies they used to address them may inform efforts to better prepare and support nurses and public health measures when facing a resurgence of COVID-19 or new pandemics. This study aimed to explore the experiences of nurses caring for people with suspected or diagnosed COVID-19 in Hong Kong.

Design A qualitative study was conducted using individual, semistructured interviews. All interviews were audio-recorded and transcribed verbatim for thematic analysis.

Setting Participants were recruited from acute hospitals and a public health department in Hong Kong from June 2020 to August 2020.

Participants A purposive sample of registered nurses $(\mathrm{N}=39)$ caring for people with COVID-19 in Hong Kong were recruited.

Results Two-thirds of the nurses had a master's degree and over a third had 6-10 years of nursing experience. Around $40 \%$ of the nurses cared for people with COVID-19 in isolation wards and a quarter performed COVID-19related work for 31-40 hours/week. Most (90\%) had training in COVID-19 and three-quarters had experience of working in infection control teams. Six key themes emerged: confronting resource shortages; changes in usual nursing responsibilities and care modes; maintaining physical and mental health; need for effective and timely responses from relevant local authorities; role of the community in public health protection and management; and advanced pandemic preparedness.

Conclusions Our study found that nurses possessed resilience, self-care and adaptability when confronting resource shortages, changing nursing protocols, and physical and mental health threats during the COVID-19 pandemic. However, coordinated support from the clinical environment, local authorities and community, and advanced preparedness would likely improve nursing responses to future pandemics.

\section{BACKGROUND}

In late December 2019, a number of cases of pneumonia with unknown aetiology were reported in Wuhan, China. ${ }^{1}$ The first

\section{STRENGTHS AND LIMITATIONS OF THIS STUDY}

$\Rightarrow$ This is the first qualitative study to explore the experiences of registered nurses caring for people with suspected or confirmed COVID-19 in Hong Kong.

$\Rightarrow$ The study recruited 39 registered nurses from acute hospitals and a public health department across Hong Kong to reflect diverse perspectives.

$\Rightarrow$ Purposive sampling was used to recruit participants and the findings may not reflect the experiences of all nurses.

confirmed case of COVID-19 was reported in Hong Kong on 23 January 2020. ${ }^{2}$ Subsequently, the WHO declared the COVID-19 outbreak a pandemic on 11 March 2020 as a result of its rapid global spread. ${ }^{3}$ By 16 March 2020, more than 120 million cases of COVID-19 had been confirmed worldwide, resulting in more than 2 million deaths across 192 countries/regions. ${ }^{4}$ In Hong Kong, to date over 11000 cases have been confirmed, with more than 200 deaths. $^{2}$

Healthcare systems globally are facing tremendous challenges in combating the COVID-19 pandemic. Unprecedented measures have been taken by healthcare authorities to cope with potential surges in patients. For instance, general wards have been set up as isolation wards, and nurses and other front-line healthcare workers without infectious diseases expertise have stepped up to provide care for people with COVID-19. To alleviate the risk of viral spread and crossinfections among patients and staff within clinical settings, further measures, such as suspending elective surgeries, have been taken. $^{67}$

Nurses are working relentlessly on the front line of care, with a large number, irrespective of specialty, deployed to manage people with COVID-19. This includes nurses with limited infectious diseases expertise suddenly working in entirely unfamiliar and stressful environments, exposing them to significant 
risk. ${ }^{8}$ By the end of October 2020, more than 1500 nurses worldwide had lost their lives to the virus. ${ }^{9}$ Regardless, nurses have consistently demonstrated commitment and compassion, often in the face of misinformation and adversity. ${ }^{10}$ A systematic review of 13 qualitative studies of nurses' experiences during respiratory pandemics, including COVID-19, found that although their professional sense of duty, dedication, self-sacrifice and collegiality were intensified, concerns arose about their personal and family safety, and physical and emotional vulnerability. ${ }^{11}$

COVID-19 is known to exert a significant psychological toll on healthcare workers as well as the general public. ${ }^{12}$ In 2003, the severe acute respiratory syndrome (SARS) epidemic in Hong Kong, where 22\% of all infected people were healthcare workers, eight of whom died, ${ }^{13} 14$ also revealed that healthcare workers faced extraordinary stress and mental health problems due to factors such as risk of infection, stigmatisation and understaffing. ${ }^{15} 16$ Qualitative studies of nurses responding to the COVID-19 pandemic in Turkey, ${ }^{17} \operatorname{Iran}^{18}$ and Spain ${ }^{19}$ have reported adverse psychosocial impacts, including anxiety and depressive symptoms, from the resulting high mortality and unpredictability. A study of 20 Chinese front-line nurses showed that during the COVID-19 pandemic, positive and negative emotions interweaved and coexisted, ${ }^{20}$ with negative ones being dominant initially and positive ones appearing gradually, with self-coping styles and psychological growth playing an imperative role in maintaining nurses' mental health.

Despite the commitment and resilience of nurses, nursing responses to the COVID-19 pandemic face numerous barriers. A qualitative systematic review of nine studies found that nurses' barriers to caring for people with COVID-19 included the unpredictability of nursing roles, lack of support, family concerns and psychological distress. ${ }^{21}$ Pivotal aspects to aid nursing's contribution to fighting COVID-19 include the role of nurses in health education, especially infection prevention and surveillance, the implementation of appropriate precautionary measures in nursing homes and protection of patients with long-term illnesses who are prone to infection, and the provision of personal protective equipment (PPE), ${ }^{22}$ as well as reasonable work schedules, effective communication, psychological support and intensive training for those who lack experience in managing infectious diseases. ${ }^{5}$

While critical aspects of nursing care may be largely universal, nurses' experiences are likely to differ between regions and countries due to the significant variations in the impact of the pandemic. ${ }^{23}$ In Hong Kong, the COVID-19 response, led by strong community mobilisation in mask-wearing, personal hygiene and social distancing as a remnant of the SARS epidemic, and a high receptiveness to antiepidemic measures, including border control and contact tracing, may be unique compared with other developed regions. ${ }^{24}{ }^{25}$ However, we have only found one published study on the experiences of Hong
Kong school nurses during the COVID-19 pandemic. ${ }^{26}$ No study has reported the experiences of nurses caring for people with COVID-19 in Hong Kong.

\section{METHODS \\ Aims}

This study aimed to explore the experiences of registered nurses caring for people with suspected or diagnosed COVID-19 during the first 6 months of the ongoing global pandemic, including the challenges they encountered and the strategies they adopted to address them, and their views about the preparation and support of nurses and public health measures regarding future pandemics.

\section{Participants}

A purposive sample of 39 registered nurses with the following inclusion criteria were recruited: (1) experience of providing direct nursing care to people with suspected and/or confirmed COVID-19 in hospital or other clinical settings; (2) able to communicate in Cantonese; and (3) consented to participate in the study. Participants were recruited from acute hospitals and a public health department in Hong Kong to allow for a breadth of diverse experiences of nurses at the front line of the COVID-19 pandemic.

Prior to data collection, participants were given a full explanation of the study objectives and procedures and informed of their right to agree or refuse to participate or withdraw from the study at any time, following which written informed consent was obtained. All data were anonymous, used for research purposes only and kept strictly confidential in a locked cabinet or via encryption, with access given only to the research team.

\section{Data collection}

A semistructured interview guide was developed (online supplemental file 1). Eligible participants were interviewed by phone by a research assistant experienced in conducting qualitative interviews. Participants were invited to share their experiences of caring for people with COVID-19, challenges encountered, strategies adopted to address them, and views about the preparation and support of nurses and public health measures regarding future pandemics. Each interview was conducted in Cantonese and audio-recorded. Participants' demographic and work characteristics were also collected, including age, sex, marital status, number of children, educational level, years of work experience, professional position, department/ward, time spent caring for people with COVID-19, previous infection control experience, training on COVID-19, wash-out period, living situation and sleep quality and quantity.

\section{Data analysis}

Data were transcribed verbatim from the audio recordings by the third author (RS), who was fluent in Cantonese and English. The third author then analysed 
all transcripts thematically based on the six phases of thematic analysis outlined by Braun and Clarke. ${ }^{27}$ The transcripts were coded and grouped under themes and subthemes according to the aims of the study. The themes were considered carefully in relation to the overall data set, and consensus was reached through checking and discussion by team members, ensuring that the most representative themes and subthemes were chosen. Illustrative quotes were translated from Cantonese to English to support the themes and subthemes found.

To ensure qualitative rigour and trustworthiness, data were collected until data saturation was achieved, indicating that adequately rich data had been collected and we would be unlikely to find any new information on continued data collection. A transparent audit trail was maintained through written reflexive notes and clear documentation of all coding decisions.

\section{Patient and public involvement}

As this study focused on the experiences of nurses caring for people with COVID-19, patients or the public were not involved.

\section{RESULTS}

Thirty-nine registered nurses were recruited and participated throughout the study. Interviews were conducted from June 2020 to August 2020 and lasted a median of 60 (range 35-89) min. Table 1 summarises participants' demographic and work characteristics. Six key themes emerged.

\section{Theme 1: confronting resource shortages}

All participants emphasised a shortage of resources, invariably PPE, isolation wards and beds, as the biggest challenge in the care of people with suspected or confirmed COVID-19. Due to high case loads, particularly from a large number of imported cases, participants encountered problems securing appropriate negative pressure and isolation rooms for high-risk individuals and procedures, and a lack of basic PPE, including face shields, masks and gloves. They commonly expressed feelings of frustration and powerlessness in their ability to care properly for patients:

PPE is a very basic need. How can I properly take care of patients when I cannot even protect myself? (Participant 15, male, registered nurse (RN))

As a result of a lack of space in hospitals, participants described having no choice but to ask suspected individuals to wait outside in open areas or in corridors in the accident and emergency department prior to COVID-19 testing. They stressed the importance of being adaptable and having hospital measures in place to address flexibly envisaged increases in patient numbers, for instance setting up enhanced surveillance wards and converting medical wards to isolation wards. At the same time, most participants acknowledged the limitations posed by such
Table 1 Demographic and work characteristics of the 39 interviewed nurses

\begin{tabular}{|c|c|}
\hline Characteristics & n (\%) \\
\hline \multicolumn{2}{|l|}{ Age (years) } \\
\hline $20-29$ & $12(30.8)$ \\
\hline $30-39$ & $17(43.6)$ \\
\hline $40-49$ & $5(12.8)$ \\
\hline $50-59$ & $5(12.8)$ \\
\hline \multicolumn{2}{|l|}{ Sex } \\
\hline Male & $12(30.8)$ \\
\hline Female & $27(69.2)$ \\
\hline \multicolumn{2}{|l|}{ Marital status } \\
\hline Single & $21(53.8)$ \\
\hline Married & $18(46.2)$ \\
\hline \multicolumn{2}{|l|}{ Children } \\
\hline One & $3(7.7)$ \\
\hline More than one & 7 (17.9) \\
\hline None & $29(74.4)$ \\
\hline \multicolumn{2}{|l|}{ Education } \\
\hline Bachelor's degree & $10(25.6)$ \\
\hline Master's degree & $26(66.7)$ \\
\hline Doctoral degree & $3(7.7)$ \\
\hline \multicolumn{2}{|l|}{ Years of nursing experience } \\
\hline $1-5$ & $11(28.2)$ \\
\hline $6-10$ & $14(35.9)$ \\
\hline $11-15$ & $4(10.3)$ \\
\hline$>15$ & $10(25.6)$ \\
\hline \multicolumn{2}{|l|}{ Position } \\
\hline Registered nurse & $23(58.9)$ \\
\hline Advanced practice nurse & $8(20.5)$ \\
\hline Senior nursing officer & $1(2.6)$ \\
\hline Ward manager & $3(7.7)$ \\
\hline Nurse consultant & $3(7.7)$ \\
\hline Department operations manager & $1(2.6)$ \\
\hline \multicolumn{2}{|c|}{ Ward worked when caring for people with COVID-19 } \\
\hline Isolation ward* & $16(41.0)$ \\
\hline Intensive care unit $†$ & $7(20.6)$ \\
\hline Accident and emergency departmentł & $8(23.5)$ \\
\hline Medical and geriatric ward§ & $1(2.9)$ \\
\hline Paediatric ward & $5(14.7)$ \\
\hline Other roles & $2(5.1)$ \\
\hline \multicolumn{2}{|c|}{ Time spent doing COVID-19-related care or work (hours/week) } \\
\hline $1-10$ & $6(15.4)$ \\
\hline $11-20$ & $9(23.1)$ \\
\hline $21-30$ & $2(5.1)$ \\
\hline $31-40$ & $10(25.6)$ \\
\hline$>40$ & $9(23.1)$ \\
\hline Difficult to measure & $3(7.7)$ \\
\hline \multicolumn{2}{|c|}{ Time spent doing COVID-19-related care or work (months) } \\
\hline
\end{tabular}




\section{Table 1 Continued}

\begin{tabular}{|c|c|}
\hline Characteristics & n (\%) \\
\hline $1-2$ & $8(20.5)$ \\
\hline $3-4$ & $10(25.6)$ \\
\hline $5-6$ & $20(51.3)$ \\
\hline $7-8$ & $1(2.6)$ \\
\hline \multicolumn{2}{|c|}{ Previous infection control team experience? } \\
\hline Yes & $29(74.4)$ \\
\hline No & $10(25.6)$ \\
\hline \multicolumn{2}{|c|}{ Received COVID-19-related training? } \\
\hline Yes & $35(89.7)$ \\
\hline No & $4(10.3)$ \\
\hline \multicolumn{2}{|c|}{ Have washout period? ${ }^{\star \star}$} \\
\hline Yes & $6(15.4)$ \\
\hline No & $31(79.5)$ \\
\hline Not applicable & $2(5.1)$ \\
\hline \multicolumn{2}{|c|}{ Living situation during care of people with COVID-19 } \\
\hline Hotel & $19(48.7)$ \\
\hline Flat rental & $3(7.7)$ \\
\hline No change & $17(43.6)$ \\
\hline \multicolumn{2}{|c|}{ Sleep quality during care of people with COVID-19 } \\
\hline Good & $3(7.7)$ \\
\hline Normal & $26(66.7)$ \\
\hline Poor & $10(25.6)$ \\
\hline \multicolumn{2}{|c|}{ Sleep quantity during care of people with COVID-19 } \\
\hline Increased & $3(7.7)$ \\
\hline No change & 32 (82.1) \\
\hline Decreased & 4 (10.3) \\
\hline \multicolumn{2}{|c|}{$\begin{array}{l}\text { *Isolation wards are rooms equipped with negative pressure air } \\
\text { extraction systems to prevent the spread of pathogens, including from } \\
\text { people with suspected or diagnosed COVID-19. A full set of personal } \\
\text { protective equipment must be worn when entering these wards. } \\
\text { †Intensive care units are dedicated wards for the provision of intensive } \\
\text { care, including to critically ill people with COVID-19. } \\
\text { †Accident and emergency departments provide services for critically } \\
\text { ill or injured people. People suspected with COVID-19 symptoms are } \\
\text { commonly identified through enhanced surveillance in these facilities. } \\
\text { \$Medical and geriatric wards target the care of elderly patients. Older } \\
\text { people admitted due to other health conditions may be identified as } \\
\text { suspected COVID-19 cases. } \\
\text { IPaediatric wards provide care to minors under } 18 \text { years old. Children } \\
\text { with suspected COVID-19 symptoms and their parents may be } \\
\text { isolated and put under surveillance in these wards. } \\
\text { **A washout period is defined as a period of time of typically } \\
2-4 \text { weeks that is taken off from work after being deployed to take care } \\
\text { of people with suspected or diagnosed COVID-19 in isolation wards } \\
\text { and before resuming previous clinical duties in other wards. }\end{array}$} \\
\hline
\end{tabular}

measures, such as their likely failure to provide adequate isolation environments:

As there weren't enough isolation wards, some general wards were converted to isolation wards...The problem is that the setting inside is not the same [in the converted wards]...we need separate places for entering and leaving in isolation wards as we need to de-gown before we can go out [of the ward] ...patient flow is affected. (Participant 19, female, RN)

They further revealed that PPE standards had been frequently downgraded in response to difficulties faced by hospitals acquiring PPE stock. While they understood the rationale for this, participants felt that health worker and patient safety was being compromised. Most participants mentioned using their own initiative to enhance personal precautions, such as minimising physical contact with patients and ensuring efficient use of equipment:

To better manage the PPE resources that we have, I try to talk to patients using a walkie talkie from outside the [isolation] room instead of in person... When I wear PPE and go inside the [isolation] room, I try to be more careful and plan what I may need well so I don't need to leave the room to get anything and waste a set of PPE. (Participant 28, female, nurse consultant)

\section{Theme 2: changes in usual nursing responsibilities and care modes}

Adapting to new and erratic procedural guidelines and protocols Most participants expressed that the experiences of the SARS and swine influenza epidemics had influenced significantly hospital planning, including workflows and procedural guidelines, during the COVID-19 pandemic. However, they also mentioned the need to learn numerous new specific protocols and guidelines, including triage criteria, transport of patients to intensive care, nasopharyngeal swab (NPS) and nasopharyngeal aspirate (NPA) procedures, safe handling of test specimens, and transfer and admission of suspected or confirmed cases under quarantine. They also reiterated the importance of nurses' awareness of environmental contamination risks and abiding by infection control practices more strictly, for instance the proper use of PPE and hand hygiene:

I had to quickly learn how to take care of isolated patients...how to do infection control [well], like donning and doffing PPE and washing hands...I also had to learn to cooperate with my colleagues [to combat this pandemic]. (Participant 15, male, RN)

Participants highlighted that coping with rapidly changing guidelines and protocols posed a major challenge. They frequently needed to ensure that they remained updated on modifications to infection control policies in response to PPE shortages, requirements of FTOCC (fever, travel history, occupational exposure, contact history, clustering) risk assessment, and new knowledge on disease pathology and symptoms to inform screening, treatment and infection control:

The guidelines we need to follow keep changing every day...so when I go to work, I first need to see what changes there are, for example, which countries are on the high travel risk list...Initially, we used to use NPS for COVID-19 tests, but now, it has been changed 
to NPA...we needed to adapt to the new procedures very fast. (Participant 16, male, $\mathrm{RN}$ )

Consequently, participants emphasised the importance of rapid dissemination of evidence-based information from reliable and easily accessible sources, particularly text messaging groups with hospital and ward staff, change-ofshift meetings, and communication kits prepared by the hospital authority (HA):

We need to always keep ourselves updated, for example about PPE stocking, so we can modify our care practices... [To do this,] I usually look at emails from the hospital, our HA Chat [A secure platform for staff to send text, photo, voice, and video messages], the communication kit from HA ...There's a lot of information out there...it can be difficult to screen and see which is the most important or trustworthy. (Participant 28, female, nurse consultant)

\section{Refocusing patient care on psychological aspects and meeting} basic needs

With most people with COVID-19 admitted to hospital being asymptomatic and relatively physically healthy, participants urged a refocusing of patient on psychological well-being. They expressed the need to spend most bedside care time assisting isolated patients to manage their fluctuating emotions, and alleviating their feelings of fear, sadness and loneliness:

We need to help patients stay positive... [some patients] start crying and we need to go into the isolation room in full gear to help them calm down... They feel scared as they don't know what's going on...stressed as [there are] a lot of unknowns...need to spend some time with them. (Participant 2, female, advanced practice nurse (APN))

Some participants indicated that a lack of patient cooperation was common due to patients' lack of understanding of the virus, and stress and frustration from receiving a positive COVID-19 test result. They asserted that effective communication with patients, including clear explanations of their possible length of stay, treatment flow and testing requirements for discharge, was vital in reassuring them and reducing their distress:

You need to have two negative COVID-19 tests with the same type of clinical specimen 24 hours apart before you can be released from isolation...A patient kept getting fluctuating results...[He] got very frustrated and said, 'Why didn't anyone tell me about this rule earlier?'...tried to leave the hospital...We called the police and he came back after talking to them... Communicating the rules and plans [to patients] early is very important...cannot assume they know why they are in hospital. (Participant 6 , female, APN)

Besides psychological care, participants conveyed that meeting the basic needs of patients to ensure a more comfortable stay in hospital also constituted a major part of care. It was important for them to be flexible and receptive to the needs of patients who were isolated in hospital for long periods of time, particularly regarding their food and entertainment preferences, including internet connectivity and toiletries:

Due to prolonged hospitalisation, patients have some very simple needs, like Wi-Fi, TV...They don't want to eat hospital food...order delivery. (Participant 4, female, RN)

Some participants expressed that patient care additionally extended to family members. As close contacts, it was necessary to inform family members of their need to be quarantined, as well as regularly update them on the patient's condition and circumstances. Patients were also provided with video calling capabilities to maintain social contact:

For elderly patients [without a phone with video calling capabilities], we give them a Tablet to use so that they can video call their family members. (Participant 7 , female, ward manager)

\section{Theme 3: maintaining physical and mental health}

Tackling adverse impacts on nurses' physical and mental health Most participants revealed that they stayed in hotels rather than at their homes to keep their families safe when taking care of people with COVID-19. They disclosed strong feelings of loneliness and social isolation from their friends and families, spending large amounts of time alone to avoid potentially infecting others. Some participants also conveyed being afraid of possible social rejection by friends as a concern as they may be seen as 'dirty' due to their place of work. All of these resulted in negative thoughts and emotions in participants:

Most of us [nurses] feel very alienated from society... We try to isolate ourselves from our family members and friends...have less social interaction with people as we don't want to infect them...I feel that I don't have much social support... feel a bit depressed. (Participant 39, female, department operations manager)

Moreover, some participants expressed feeling fearful when taking care of patients, in particular during highrisk procedures, especially involving aerosols. However, at the same time, most participants felt that regardless of these negative feelings, they held a strong sense of professional responsibility to contribute to the fight against the pandemic and had a strong faith in their ability to protect themselves and others:

I found out I was pregnant while working in the isolation ward...My colleagues and family were worried about me, but I decided to continue working there...I had a friend with a similar experience and she was ok...if I wear full PPE properly and trust my skills, 
I can still do my job safely. (Participant 35, female, APN)

As a result, participants indicated working harder to ensure their physical health through effective self-care and personal protection practices, including taking greater care and alertness in infection control, and clear planning and preparation prior to conducting high-risk procedures:

When performing high-risk aerosol-generating procedures on confirmed cases, we need to be very clear on the steps we take...need to be smooth...very alert, especially during doffing of PPE, washing hands, and showering [afterwards]. (Participant 32, male, RN)

In addition, participants further highlighted that family, friends and peer support were essential in promoting their mental health, providing reassurance and alleviating their worries and anxieties:

My friends and family call me to tell me to be careful...I also have very supportive friends in the hospital who constantly ask if I need anything...I don't feel that lonely [because of them]. (Participant 6, female, APN)

\section{Importance of teamwork and a supportive practice environment}

Most participants emphasised that the support and cooperation of their team played an important role in helping them maintain their physical and mental health. With large numbers of new staff deployed from other wards, participants also expressed that it was necessary to work together to train them, and for more experienced and senior nurses to ensure their understanding of nursing interventions:

We have very good team spirit...everyone is very supportive... We use a buddy system so that when one of us is going to take care of [infected] patients, there would be another person watching you to make sure you do all the [nursing] procedures safely. (Participant 5, female, RN)

Additionally, participants emphasised the benefit of a supportive hospital environment, particularly the management team. Some participants appreciated the hospital management team for arranging staff rental allowances and subsidies, although others asserted that improved PPE and understanding of front-line staff needs were required. Most highlighted that explaining the rationale behind management decisions was imperative in reducing discontent and negative feelings among front-line staff:

Frontline staff needs to be given more information on management decisions...if they can understand why different decisions are made, they would have fewer grievances and can be more supportive. (Participant 10 , male, senior nursing officer)
Theme 4: need for effective and timely responses from relevant local authorities

Most participants described the responses of governmental departments as an important factor in ensuring adequate public health measures during the COVID-19 pandemic. While they stressed such departments had the ability to considerably enhance care provision within manageable patient case loads, some criticised the inadequate response measures of local authorities in relation to the establishment of protocols and guidelines and isolation wards, management of PPE shortages, and stepdown arrangements for non-serious cases:

Many things need to be prepared earlier, especially hospital negative pressure rooms...In my hospital, there was a time when we needed to urgently prepare the negative pressure room, but we noticed that there was some water leakage there and a little problem with the double doors of the room...these things should have been fixed earlier as we never know when there may be a sudden explosion in cases. (Participant 4, female, RN)

Most participants supported the introduction of earlier, enforced government responses to the pandemic: restrictions on travel into the city, early screening, monitoring and management of quarantined cases, active promotion of mask-wearing, and surgical mask distribution to disadvantaged groups. Some participants suggested that public health decision-makers should have sought greater involvement of front-line healthcare professionals:

More frontline [healthcare] professionals should be involved in giving input to the government...the government should listen to their suggestions...tell people to wear masks earlier, control number of people on public transport in rush hour, and disseminate more information on how people can protect themselves. (Participant 2, female, APN)

Overall, participants attributed the lack of sufficient foresight and a severe underestimation of the disease by authorities as a prominent cause of the burden faced by nurses during the pandemic.

\section{Theme 5: role of the community in public health protection and management}

Apart from the provision of direct care to infected individuals, participants mentioned the value of collective community efforts in effectively controlling infectious disease outbreaks. They emphasised the importance of promoting the proper adoption of simple personal protective and hygiene measures, namely keeping hands clean by washing them or using hand sanitisers, wearing eye goggles and masks, social distancing, and ensuring cleanliness of the surrounding environment within the community. Some participants also highlighted the need for specific measures in the Chinese cultural context, such as avoiding tea and hotpot gatherings, and using serving chopsticks during meals: 
When people started wearing masks, we noticed that the number of patients coming in with other URIs [upper respiratory infections] also reduced...clearly, such measures are very important and effective. (Participant 31, female, RN)

Consequently, participants stressed the additional role of nurses in acting as community health educators to enhance public health education and alertness. Owing to the lack of an effective vaccine or treatment for COVID-19 at the time, they also asserted that the government should take a greater role in reducing misinformation and disseminating more evidence-based information to maintain public vigilance and reduce community spread of the disease:

Now we know that the virus is spread by droplets... but people [the general public] don't have any clear information on what precautions to take against this...I always see people in the bus...touching everywhere and not being that aware. (Participant 22, female, RN)

\section{Theme 6: advanced pandemic preparedness}

More comprehensive and ongoing nursing education on outbreak management

Participants reiterated the necessity for regular and continuous infection control training of nurses, particularly regarding their psychological preparation, awareness and alertness to fight infectious disease outbreaks, and optimising their clinical skills in performing highrisk interventions, reducing cross-infection and contamination risks, and donning and doffing PPE. They also suggested the use of outbreak training simulations and experience sharing by front-line nurses to bolster such skills, and emphasised the promotion of teamwork, delegation and communication among nurses during disease outbreaks:

More nurse education on high-risk interventions, including how to reduce contact with droplets and protect ourselves, manage emotional health, perform [effective] division of labour, delegate things systematically to avoid 'overloading' ourselves, and improve teamwork is needed. (Participant 23, male, RN)

\section{Early resource planning and preparation}

Most participants emphasised the importance of early, large-scale warning and preparation of health systems and facilities to deal with infectious disease outbreaks. Although experiences in previous epidemics in Hong Kong had improved local reserves of resources, including PPE, ventilators, isolation and quarantine facilities, and negative pressure rooms, participants expressed the need to re-evaluate and update current practices. They also warned that adaptations to sudden increases in COVID-19 case numbers in Hong Kong hospitals had exposed inadequacies and risks in current measures:
HA should set up more isolation facilities in advance. They may seem useless at the time, especially if we don't have any disease outbreak for many years, but you never know when there may be a sudden outbreak.... When you convert general wards to isolation wards, you may jeopardise patient safety as they don't fit the required infection control criteria completely. (Participant 19, female, RN)

Additionally, participants recounted that the severe PPE shortages experienced at the initial stages of the pandemic had highlighted the need to enhance local PPE production knowledge and establish local production lines of high-quality PPE, particularly masks:

We need steady production of PPE in Hong Kong to cater to the sudden demand [during epidemics]... right now, even if we want to boost [PPE] production, we don't have the technology or know-how...need it in advance. (Participant 30, female, nurse consultant)

\section{DISCUSSION}

To our knowledge, this is the first qualitative study to explore registered nurses' experiences of caring for people with COVID-19 in Hong Kong. Our findings highlight the resilience and adaptability of nurses in tackling numerous obstacles in the provision of effective nursing care during the pandemic. In the face of a lack of resources, particularly PPE and isolation facilities, nurses showed initiative and creativity in adapting to and overcoming such major obstacles. This accords with strategies suggested by the WHO and the Centers for Disease Control and Prevention that have been used by healthcare workers when confronted with limited supplies, such as limiting patient contact and using the same respirator with multiple patients. ${ }^{28}$

Moreover, our findings emphasise the widespread need for and use of self-care and self-protection measures, including greater awareness of personal infection control precautions, and more careful planning and preparation by nurses caring for people with COVID-19. However, none of the nurses articulated using a clear set of guidelines regarding their own self-care and well-being; rather they used personal strategies based on their own knowledge and experience. ${ }^{29}$ The need for self-care and support was similarly reported in a qualitative study of Australian primary healthcare nurses' needs during the COVID-19 pandemic. ${ }^{30}$

Nurses were highly adaptable in prioritising, accessing and adjusting to the use of ever-changing COVID-19specific nursing care protocols and guidelines. They were also aware of and responsive to the specific needs of people with COVID-19, particularly comfort and psychological well-being, and minimising the mental health burden so prevalent in this group. ${ }^{31}$ Notably, most nurses emphasised they were able to provide psychological support as many of the patients were asymptomatic or 
had mild symptoms and were relatively physically healthy, given Hong Kong's strict policy regarding the admission of all COVID-19 cases to reduce the community spread of the virus. This is in contrast to studies from other countries such as China ${ }^{32}$ and Italy, ${ }^{33}$ where nurses faced tremendous challenges in caring for heavy workloads of seriously ill people with COVID-19.

It was apparent that clear communication was central to enhancing mutual nurse-patient understanding and assuaging any hostility arising from patients' fear, anxiety and misconceptions. Interestingly, this was most prominent regarding non-local patients, a growing population as a consequence of the international hub status of Hong Kong. Recent reports of threats or acts of violence, including physical and verbal abuse, against healthcare workers during the COVID-19 pandemic, although largely from patients' relatives, due to a lack of knowledge about COVID-19 and its treatment, and dissatisfaction with hospital policies, ${ }^{34}{ }^{35}$ illustrate the importance of good communication skills among nurses.

When confronted with their own feelings of despair and unease due to the ambiguity and unpredictability associated with COVID-19, nurses emphasised effective cooperation and teamwork strategies as means to ensure safety and reassurance among colleagues. Regardless of such negative feelings and loneliness resulting from working at the front line of the pandemic, nurses emphasised their sense of professional duty, pride and responsibility, bolstered by support of colleagues, friends and family. Consistent with this, a cross-sectional study of 325 nurses in the Philippines asserted that perceived organisational and social support were predictors of COVID-19-related anxiety in nurses. ${ }^{36}$

Our findings also revealed suggestions to improve measures for dealing with future outbreaks. Nurses wished to have their viewpoints taken into consideration in hospital management decisions about the pandemic, particularly when such decisions had consequences for nurses' safety and performance. Such an organisational response may reduce burnout and psychological distress in nurses, as reported by a recent systematic review of 13 qualitative studies. ${ }^{11}$ To allow for a more targeted and wellprepared pandemic response, our findings emphasise the need for consistent updating of nurses' infection control education and the rapid availability of sufficient reserves of resources and isolation facilities. This is supported by an online cross-sectional study of 637 primary healthcare nurses in Australia, which identified seven key categories of perceived nurse support needed to provide quality clinical care during the pandemic: PPE, communication, funding, industrial issues, self-care, workplace factors and valuing nurses. ${ }^{30} \mathrm{~A}$ cross-sectional study of 261 front-line nurses in the Philippines highlighted the importance of organisational measures to support nurses' mental health and reduce fear, including social support, psychological support services, COVID-19-related training, and accurate and regular information and updates. ${ }^{37}$ Interestingly, while most studies emphasised the importance of psychological support and coping strategies to alleviate fear and distress among nurses, ${ }^{30} 31$ 36-38 our study found that nurses appeared to focus largely on material or nursing care-specific support, particularly improved PPE management and training.

Finally, it was emphasised by participants that while nurses can assist in the control of pandemics, the collective and concerted contributions of a well-educated and wellinformed community are essential. Our findings support the need for multifaceted responses involving multiple stakeholders, including nurses as well as policy makers, hospital administrators and the local community. ${ }^{29}$

\section{Limitations}

The limitations of this study are that we used a purposive sample of registered nurses in acute hospitals and a public health department, and our findings may not reflect the experiences of all nurses in these settings. In addition, all the recruited participants were nurses from Hong Kong, so this study only explored the situation of the pandemic in the region. Also, as our findings represent the experiences of nurses at the time of the interviews, they are unable to address the dynamic nature of the global pandemic. Finally, to avoid close contact with healthcare workers providing direct care to people with COVID-19, we had to conduct interviews by phone.

\section{Implications for research and practice}

Findings from this study may help to optimise nursing responses to future pandemics by improving the preparedness of nurses through the provision of appropriate education and training regarding responses against novel infectious diseases, PPE management during shortages, communication skills and psychological patient care. In addition, policy makers and managers should regularly re-evaluate contingency plans regarding PPE and ensure the involvement of nurses.

Longitudinal studies of the experiences of nurses caring for people over the course of COVID-19 are recommended. These may include robust measures of stress, self-efficacy and personal control, for example.

\section{CONCLUSIONS}

Nurses have shown remarkable resilience and adaptability, despite resource shortages and mental and physical health threats, when caring for people with COVID-19. However, nurses need appropriate support from peers, managers, policy makers and the local community to effectively prepare for and manage such pandemics. The findings of this study may help inform future nursing practice, education and policy making to shape and strengthen the response to global infectious disease outbreaks.

Acknowledgements We would like to thank all nurse participants for consenting to take part in this study.

Contributors JPCC and SHSL conceived, designed and supervised the study. CHYL and SKYL led the data collection process. RS and CHYL processed and analysed 
the data. JPCC, SHSL, RS, CHYL and DT contributed to drafting the full article. All authors read and approved the final manuscript.

Funding The authors have not declared a specific grant for this research from any funding agency in the public, commercial or not-for-profit sectors.

Competing interests None declared.

Patient consent for publication Not required.

Ethics approval This study received ethical approval from the Survey and Behavioural Research Ethics Committee of The Chinese University of Hong Kong (reference no: SBRE-19-594) and followed the principles of the Declaration of Helsinki.

Provenance and peer review Not commissioned; externally peer reviewed.

Data availability statement Data are available upon reasonable request. Data supporting the findings of this study are available from the corresponding author upon reasonable request.

Supplemental material This content has been supplied by the author(s). It has not been vetted by BMJ Publishing Group Limited (BMJ) and may not have been peer-reviewed. Any opinions or recommendations discussed are solely those of the author(s) and are not endorsed by BMJ. BMJ disclaims all liability and responsibility arising from any reliance placed on the content. Where the content includes any translated material, BMJ does not warrant the accuracy and reliability of the translations (including but not limited to local regulations, clinical guidelines, terminology, drug names and drug dosages), and is not responsible for any error and/or omissions arising from translation and adaptation or otherwise.

Open access This is an open access article distributed in accordance with the Creative Commons Attribution Non Commercial (CC BY-NC 4.0) license, which permits others to distribute, remix, adapt, build upon this work non-commercially, and license their derivative works on different terms, provided the original work is properly cited, appropriate credit is given, any changes made indicated, and the use is non-commercial. See: http://creativecommons.org/licenses/by-nc/4.0/.

\section{ORCID iDs}

Janita Pak Chun Chau http://orcid.org/0000-0002-3750-7396

Suzanne Hoi Shan Lo http://orcid.org/0000-0002-9970-0642

\section{REFERENCES}

1 Zhu N, Zhang D, Wang W, et al. A novel coronavirus from patients with pneumonia in China, 2019. N Engl J Med 2020;382:727-33.

2 Centre for Health Protection. Coronavirus disease (COVID-19) in HK [online], 2021. Available: https://chp-dashboard.geodata.gov.hk/ covid-19/en.html [Accessed 16 Mar 2021]

3 World Health Organization. Listings of WHO's response to COVID-19 [online], 2020. Available: https://www.who.int/news-room/detail/2906-2020-covidtimeline [Accessed 19 Oct 2020].

4 Johns Hopkins University \& Medicine. COVID-19 dashboard by the center for systems science and engineering (CSSE) at Johns Hopkins University (JHU) [online], 2021. Available: https://coronavirus.jhu.edu/ map.html [Accessed 16 Mar 2021].

5 Liu Q, Luo D, Haase JE, et al. The experiences of health-care providers during the COVID-19 crisis in China: a qualitative study. Lancet Glob Health 2020;8:e790-8.

6 Phillips MR, Chang Y, Zura RD, et al. Impact of COVID-19 on orthopaedic care: a call for nonoperative management. Ther Adv Musculoskelet Dis 2020;12:1759720x20934276.

7 Teoh JY-C, Ong WLK, Gonzalez-Padilla D, et al. A global survey on the impact of COVID-19 on urological services. Eur Urol 2020;78:265-75.

8 Catton H. Global challenges in health and health care for nurses and midwives everywhere. Int Nurs Rev 2020;67:4-6.

9 International Council of Nurses. ICN confirms 1,500 nurses have died from COVID-19 in 44 countries and estimates that healthcare worker COVID-19 fatalities worldwide could be more than 20,000 [online], 2020. Available: https://www.icn.ch/news/icn-confirms-1500-nurseshave-died-covid-19-44-countries-and-estimates-healthcare-workercovid [Accessed 13 Nov 2020].

10 Smith GD, Ng F, Ho Cheung Li W. COVID-19: emerging compassion, courage and resilience in the face of misinformation and adversity. J Clin Nurs 2020;29:1425-8.

11 Fernandez R, Lord H, Halcomb E, et al. Implications for COVID-19: a systematic review of nurses' experiences of working in acute care hospital settings during a respiratory pandemic. Int J Nurs Stud 2020;111:103637.
12 Chew NWS, Lee GKH, Tan BYQ, et al. A multinational, multicentre study on the psychological outcomes and associated physical symptoms amongst healthcare workers during COVID-19 outbreak. Brain Behav Immun 2020;88:559-65.

$13 \mathrm{Hsin} \mathrm{DH}-\mathrm{C}$, Macer DRJ. Heroes of SARS: professional roles and ethics of health care workers. J Infect 2004;49:210-5.

14 Hung LS. The SARS epidemic in Hong Kong: what lessons have we learned? J R Soc Med 2003;96:374-8.

15 Grace SL, Hershenfield K, Robertson E, et al. The occupational and psychosocial impact of SARS on academic physicians in three affected hospitals. Psychosomatics 2005;46:385-91.

16 Tam CWC, Pang EPF, Lam LCW, et al. Severe acute respiratory syndrome (SARS) in Hong Kong in 2003: stress and psychological impact among frontline healthcare workers. Psychol Med 2004:34:1197-204.

17 Kackin O, Ciydem E, Aci OS, et al. Experiences and psychosocial problems of nurses caring for patients diagnosed with COVID-19 in turkey: a qualitative study. Int J Soc Psychiatry 2021;67:158-67.

18 Galehdar N, Kamran A, Toulabi T, et al. Exploring nurses' experiences of psychological distress during care of patients with COVID-19: a qualitative study. BMC Psychiatry 2020;20:489.

19 Fernández-Castillo R-J, González-Caro M-D, Fernández-García E. Intensive care nurses' experiences during the COVID-19 pandemic: a qualitative study. Nurs Crit Care 2021 doi:10.1111/nicc.12589

20 Sun N, Wei L, Shi S, et al. A qualitative study on the psychological experience of caregivers of COVID-19 patients. Am J Infect Control 2020;48:592-8.

21 Joo JY, Liu MF. Nurses' barriers to caring for patients with COVID-19: a qualitative systematic review. Int Nurs Rev 2021;68:202-13.

22 Chen S-C, Lai Y-H, Tsay S-L. Nursing perspectives on the impacts of COVID-19. J Nurs Res 2020;28:e85.

23 Yoo JY, Dutra SVO, Fanfan D, et al. Comparative analysis of COVID-19 guidelines from six countries: a qualitative study on the US, China, South Korea, the UK, Brazil, and Haiti. BMC Public Health 2020;20:1853.

24 Wan K-M, Ka-Ki Ho L, Wong NWM, et al. Fighting COVID-19 in Hong Kong: the effects of community and social mobilization. World Dev 2020;134:105055.

25 Lam HY, Lam TS, Wong CH, et al. The epidemiology of COVID-19 cases and the successful containment strategy in Hong KongJanuary to May 2020. Int J Infect Dis 2020;98:51-8.

26 Lee RLT, West S, Tang ACY, et al. A qualitative exploration of the experiences of school nurses during COVID-19 pandemic as the frontline primary health care professionals. Nurs Outlook 2021;69:399-408.

27 Braun V, Clarke V. Using thematic analysis in psychology. Qual Res Psychol 2006;3:77-101.

28 Mahmood SU, Crimbly F, Khan S, et al. Strategies for rational use of personal protective equipment (PPE) among healthcare providers during the COVID-19 crisis. Cureus 2020;12:e8248.

29 Alharbi J, Jackson D, Usher K. The potential for COVID-19 to contribute to compassion fatigue in critical care nurses. J Clin Nurs 2020;29:2762-4.

30 Halcomb E, Williams A, Ashley C, et al. The support needs of Australian primary health care nurses during the COVID-19 pandemic. J Nurs Manag 2020;28:1553-60.

31 Zhou X, Snoswell CL, Harding LE, et al. The role of telehealth in reducing the mental health burden from COVID-19. Telemed J E Health 2020;26:377-9.

32 Liu Y-E, Zhai Z-C, Han Y-H, et al. Experiences of front-line nurses combating coronavirus disease-2019 in China: a qualitative analysis. Public Health Nurs 2020;37:757-63.

33 Lasalvia A, Bonetto C, Porru S, et al. Psychological impact of COVID-19 pandemic on healthcare workers in a highly burdened area of north-east Italy. Epidemiol Psychiatr Sci 2020;30:e1.

34 Ghareeb NS, El-Shafei DA, Eladl AM. Workplace violence among healthcare workers during COVID-19 pandemic in a Jordanian governmental hospital: the tip of the iceberg. Environ Sci Pollut Res Int 2021:1-9.

35 Bhatti OA, Rauf $\mathrm{H}$, Aziz N, et al. Violence against healthcare workers during the COVID-19 pandemic: a review of incidents from a lowermiddle-income country. Ann Glob Health 2021;87:41.

36 Labrague LJ, De Los Santos JAA. COVID-19 anxiety among frontline nurses: predictive role of organisational support, personal resilience and social support. J Nurs Manag 2020;28:1653-61.

37 Labrague LJ, de Los Santos JAA. Fear of COVID-19, psychological distress, work satisfaction and turnover intention among frontline nurses. J Nurs Manag 2021;29:395-403.

38 Huang L, Lei W, Xu F, et al. Emotional responses and coping strategies in nurses and nursing students during Covid-19 outbreak: a comparative study. PLoS One 2020;15:e0237303. 